\title{
BINOMIAL EDGE IDEALS AND BOUNDS FOR THEIR REGULARITY
}

\author{
ARVIND KUMAR
}

\begin{abstract}
Let $G$ be a simple graph on $n$ vertices and $J_{G}$ denote the corresponding binomial edge ideal in $S=K\left[x_{1}, \ldots, x_{n}, y_{1}, \ldots, y_{n}\right]$. We prove that the Castelnuovo-Mumford regularity of $J_{G}$ is bounded above by $c(G)+1$, when $G$ is a quasi-block graph or semi-block graph. We give another proof of Saeedi Madani-Kiani regularity upper bound conjecture for chordal graphs. We obtain the regularity of binomial edge ideals of Jahangir graphs. Later, we establish a sufficient condition for Hibi-Matsuda conjecture to be true.
\end{abstract}

\section{INTRODUCTION}

Let $G$ be a simple graph on $[n]$ and $S=K\left[x_{1}, \ldots, x_{n}, y_{1}, \ldots, y_{n}\right]$, where $K$ is a field. The binomial edge ideal of the graph $G, J_{G}=\left(x_{i} y_{j}-x_{j} y_{i}:\{i, j\} \in E(G), i<j\right)$, was introduced by Herzog et al. in [9] and independently by Ohtani in [21]. Since then researchers have been trying to study the algebraic invariants of $J_{G}$ in terms of the combinatorial invariants of $G$. In [5, 9, 13, 15, 19, 23, 25, 26, the authors have established connections between homological invariants such as depth, codimension, Betti numbers and Castelnuovo-Mumford regularity of $J_{G}$ with certain combinatorial invariants associated with the graph $G$. The study of Castelnuovo-Mumford regularity of binomial edge ideals has attracted a lot of attention in the recent past due to its algebraic and geometric importance. In [19, Theorem 1.1], Matsuda and Murai proved that for any graph $G$ on $[n], l(G) \leq \operatorname{reg}\left(S / J_{G}\right) \leq n-1$, where $l(G)$ is the length of a longest induced path in $G$. In the same article, they conjectured that $\operatorname{reg}\left(S / J_{G}\right)=n-1$ if and only if $G=P_{n}$. This conjecture was settled in affirmative by Kiani and Saeedi Madani in [15]. For a graph $G$, let $c(G)$ denote the number of maximal cliques in $G$. If $G$ is a closed graph, i.e., the generators of $J_{G}$ are a Gröbner basis with respect to lexicographic order induced by $x_{1}>\cdots>x_{n}>y_{1}>\cdots>y_{n}$, then Saeedi Madani and Kiani [25] proved that $\operatorname{reg}\left(S / J_{G}\right) \leq c(G)$. In [26], the following conjecture was proposed.

Conjecture 1.1. Let $G$ be a graph on $[n]$. Then, $\operatorname{reg}\left(S / J_{G}\right) \leq c(G)$.

In [7], Ene and Zarojanu proved the conjecture for block graphs. In [12], Jayanthan and Kumar proved the conjecture for $k$-fan graph of the complete graph. In [24], Rouzbahani Malayeri et al. proved the conjecture for the class of chordal graphs. Recently, in [14], Kahle and Krüsemann proved the conjecture for cographs. In the third section, we prove Saeedi

Key words and phrases. Binomial edge ideal, Castelnuovo-Mumford regularity, Chordal graph, Quasiblock graph, Semi-block graph, h-polynomial.

Mathematics Subject Classification: 13D02, 05E40. 
Madani-Kiani conjecture for some classes of non-chordal graphs. We prove Conjecture 1.1 for the class of quasi-block graphs (see Sect. 3 for the definition). Indeed, we give an example of a quasi-block graph to show that the upper bound is tight. Then, we prove Conjecture 1.1 for semi-block graphs (see Sect. 3 for the definition). We then give another proof of Conjecture 1.1 for the class of chordal graphs. Also, we provide a sufficient condition for chordal graphs so that the inequality is strict in Conjecture 1.1.

In the fourth section, we obtain the regularity of binomial edge ideals of Jahangir graphs (see Sect. 4 for the definition). To compute the regularity of binomial edge ideals of Jahangir graphs, we use [6, Theorem 2.1], which is a recent result due to Ene, Rinaldo and Terai.

In [11, Hibi and Matsuda studied the regularity of binomial edge ideals of graphs from the algebraic perspective and conjectured that the regularity is bounded above by the degree of the $h$-polynomial of $S / J_{G}$.

Conjecture 1.2. [11, Conjecture 0.1] Let $G$ be a graph on $[n]$. Then, $\operatorname{reg}\left(S / J_{G}\right) \leq \operatorname{deg} h_{S / J_{G}}(t)$.

In the last section, we obtain a sufficient condition for Conjecture 1.2 to be true. Recently, in [14, Kahle and Krüsemann found a counterexample to Conjecture 1.2. We give another example to show that Conjecture 1.2 is not true in general, even for chordal graphs.

\section{Preliminaries}

In this section, we recall some notation and fundamental results on graphs and the corresponding binomial edge ideals which are used throughout this paper.

Let $G$ be a finite simple graph with vertex set $V(G)$ and edge set $E(G)$. For $A \subseteq V(G)$, $G[A]$ denotes the induced subgraph of $G$ on the vertex set $A$, i.e., for $i, j \in A,\{i, j\} \in E(G[A])$ if and only if $\{i, j\} \in E(G)$. For a vertex $v, G \backslash v$ denotes the induced subgraph of $G$ on the vertex set $V(G) \backslash\{v\}$. A vertex $v \in V(G)$ is said to be a cut vertex if $G \backslash v$ has more connected components than $G$. A subset $U$ of $V(G)$ is said to be a clique if $G[U]$ is a complete graph. For $v \in V(G)$, let $\operatorname{cdeg}_{G}(v)$ denote the number of maximal cliques which contains $v$. We say that $G$ is $k$-vertex connected, if $k<|V(G)|$ and for every subset $S \subset V(G)$ such that $|S|<k$, the induced subgraph $G[V(G) \backslash S]$ is connected. The vertex connectivity of $G$, denoted by $\kappa(G)$, is defined as the maximum integer $k$ such that $\mathrm{G}$ is $k$-vertex connected.

A simplicial complex $\Delta$ on the vertex set $[n]$ is a collection of subsets of $[n]$ such that: $(i)$ $\{v\} \in \Delta$ for all $v \in[n]$; (ii) $F \in \Delta$ and $G \subseteq F$ implies $G \in \Delta$. An element $F \in \Delta$ is called a face of $\Delta$. A maximal face of $\Delta$ with respect to inclusion is called a facet of $\Delta$. A facet $F$ of $\Delta$ is called a leaf, if either $F$ is the only facet, or else there exists a facet $G$ such that for each facet $H$ of $\Delta$ with $H \neq F, H \cap F \subsetneq G \cap F$. A vertex $v$ is said to be a free vertex (simplicial vertex) if it belongs to exactly one facet of $\Delta(G)$. Each leaf $F$ has at least one free vertex.

The simplicial complex $\Delta$ is called a quasi-forest, if its facets can be ordered $F_{1}, \ldots, F_{s}$ such that for all $i>1$, the facet $F_{i}$ is a leaf of the simplicial complex with facets $F_{1}, \ldots, F_{i-1}$. 
Such an order of the facets is called a leaf order. A connected quasi-forest is called a quasitree. The collection of all cliques of a graph $G$ form a simplicial complex which is called clique complex of $G$ and is denoted by $\Delta(G)$. Its facets are the maximal cliques of $G$. The clique number of a graph $G$, denoted by $\omega(G)$, is the maximum size of the maximal cliques of $G$.

A vertex $v$ is said to be an internal vertex, if $v$ is not a free vertex. Let $\operatorname{iv}(G)$ denote the number of internal vertices of $G$. The neighborhood of a vertex $v$, denoted by $N_{G}(v)$, is defined as $\{u \in V(G):\{u, v\} \in E(G)\}$. For a vertex $v, G_{v}$ is the graph on the vertex set $V(G)$ and edge set $E\left(G_{v}\right)=E(G) \cup\left\{\{u, w\}: u, w \in N_{G}(v)\right\}$. For $e \in E(G), G \backslash e$ is the graph on the vertex set $V(G)$ and edge set $E(G) \backslash\{e\}$.

For $T \subset[n]$, let $\bar{T}=[n] \backslash T$ and $c_{G}(T)$ denote the number of connected components of $G[\bar{T}]$. Let $G_{1}, \ldots, G_{c_{G}(T)}$ be the connected components of $G[\bar{T}]$. For each $i$, let $\tilde{G}_{i}$ denote the complete graph on $V\left(G_{i}\right)$. Set $P_{T}(G)=\left(\cup_{i \in T}\left\{x_{i}, y_{i}\right\}, J_{\tilde{G}_{1}}, \ldots, J_{\tilde{G}_{c_{G}}(T)}\right)$. In [9], it was shown by Herzog et al. that $J_{G}=\underset{T \subseteq[n]}{\bigcap} P_{T}(G)$.

The following basic property of regularity is used repeatedly in this article. We refer the reader to the book [22, Chapter 18] for more properties on regularity.

Lemma 2.1. Let $M, N$ and $P$ be finitely generated graded $S$-modules. If

$$
0 \rightarrow M \stackrel{f}{\rightarrow} N \stackrel{g}{\rightarrow} P \rightarrow 0
$$

is a short exact sequence with $f, g$ graded homomorphisms of degree zero, then

(1) $\operatorname{reg}(M) \leq \max \{\operatorname{reg}(N), \operatorname{reg}(P)+1\}$.

(2) $\operatorname{reg}(M)=\operatorname{reg}(N)$, if $\operatorname{reg}(N)>\operatorname{reg}(P)$.

\section{Saeedi Madani-Kiani Conjecture}

In this section, we prove Conjecture 1.1 for some classes of non-chordal graphs. Also, we give another proof of Conjecture 1.1 for the class of chordal graphs. We begin by recalling a lemma by Ohtani which is highly useful in computing the regularity of binomial edge ideals.

Lemma 3.1. [21, Lemma 4.8] Let $G$ be a graph. If $v$ is an internal vertex, then

$$
J_{G}=J_{G_{v}} \cap\left(\left(x_{v}, y_{v}\right)+J_{G \backslash v}\right) .
$$

Note that $J_{G_{v}}+\left(\left(x_{v}, y_{v}\right)+J_{G \backslash v}\right)=\left(x_{v}, y_{v}\right)+J_{G_{v} \backslash v}$. Therefore, we have the following short exact sequence:

$$
0 \longrightarrow \frac{S}{J_{G}} \longrightarrow \frac{S}{J_{G_{v}}} \oplus \frac{S}{\left(x_{v}, y_{v}\right)+J_{G \backslash v}} \longrightarrow \frac{S}{\left(x_{v}, y_{v}\right)+J_{G_{v} \backslash v}} \longrightarrow 0 .
$$

We first establish a connection between the number of internal vertices of $G, G_{v}$ and $G \backslash v$.

Lemma 3.2. Let $G$ be a graph on $[n]$. If $v$ is an internal vertex of $G$, then $\operatorname{iv}(G)>\operatorname{iv}\left(G_{v}\right)$ and $\operatorname{iv}(G)>\operatorname{iv}(G \backslash v)$. 
Proof. If $\operatorname{iv}\left(G_{v}\right)=0$, then $\operatorname{iv}(G)>\operatorname{iv}\left(G_{v}\right)$. Let iv $\left(G_{v}\right)=k>0$. Let $v_{1}, \ldots, v_{k}$ be the internal vertices of $G_{v}$. Note that $v$ is a free vertex of $G_{v}$. Assume that $v_{r+1}, \ldots, v_{k} \in N_{G}(v)=$ $N_{G_{v}}(v)$. For $r+1 \leq i \leq k, v_{i}$ is not a free vertex of $G_{v}$. Therefore, $v_{i}$ is not a free vertex of $G$. For $1 \leq i \leq r, v_{i}$ is an internal vertex of $G$, since $v_{i} \notin N_{G}(v)$. As $v$ is an internal vertex of $G$, we have $\operatorname{iv}(G) \geq k+1$. Let $u$ be a free vertex of $G$. In $G \backslash v, u$ is either a free vertex or an isolated vertex, i.e., $u$ is not an internal vertex of $G \backslash v$. Hence, $\operatorname{iv}(G)>\operatorname{iv}(G \backslash v)$.

So far Conjecture 1.1 has been proved only for chordal graphs, (see [24, Theorem 3.5]). There have been no attempts on non-chordal graphs. Now, we prove Conjecture 1.1 for a class of non-chordal graphs.

Let $H$ be a connected closed graph on $[n]$ such that $S / J_{H}$ is Cohen-Macaulay. By [5, Theorem 3.1], there exist integers $1=a_{1}<a_{2}<\cdots<a_{s}<a_{s+1}=n$ such that $F_{i}=\left[a_{i}, a_{i+1}\right]$, for $1 \leq i \leq s$ and $F_{1}, \ldots, F_{s}$ is a leaf order of $\Delta(H)$. Set $e=\{1, n\}$. The graph $G=H \cup\{e\}$ is called the quasi-cycle graph associated with $H$. In [20], the Hilbert series of the binomial edge ideal of quasi-cycles was studied.

Remark 3.3. Let $G$ be the quasi-cycle graph associated with a Cohen-Macaulay closed graph $H$. Let $F_{1}, \ldots, F_{s}$ be a leaf order of $\Delta(H)$. If $H \neq P_{3}$, then $\operatorname{iv}(G) \geq s$ and $\operatorname{iv}(H)=$ $s-1$. If $s=2$, then $G$ is a chordal graph. If $s>2$, then $G$ has an induced cycle of length $s+1$ on the vertex set $\left\{a_{1}, a_{2}, \ldots, a_{s}, a_{s+1}\right\}$. Thus, for $s>2, G$ is not a chordal graph.

Example 3.4. Let $H$ and $G$ be the graphs shown in the figure below. It can be observed that $H$ is a Cohen-Macaulay closed graph and $G$ is a quasi-cycle graph associated with $H$.

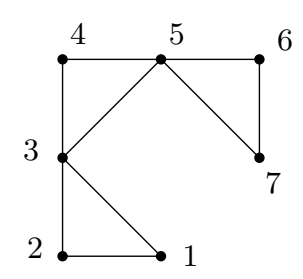

H

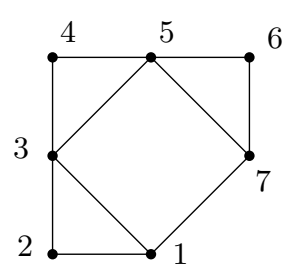

G

For a graph $G$, a maximal subgraph of $G$ without a cut vertex is called a block of $G$. A graph $G$ is said to be a block graph if each block of $G$ is a clique. A block $B$ of a graph $G$ is called a quasi-block if $B$ is a quasi-cycle other than $K_{3}$.

Definition 3.5. A graph $G$ is said to be a quasi-block graph if $G$ satisfies the following:

(1) Each block of $G$ is either a clique or a quasi-block.

(2) If $v$ is an internal vertex of a quasi-block $B$, then for any $u \in N_{G}(v) \backslash V(B), u$ is not an internal vertex of any block.

One can note that a quasi-block graph need not be a chordal graph. We denote by qc $(G)$, the number of quasi-blocks in $G$. 
Remark 3.6. If $G$ is a connected quasi-block graph and $v$ is an internal vertex of $G$, then $G \backslash v$ is a quasi-block graph. If $B$ is a quasi-block of $G$ and $v \in V(B)$ is an internal vertex of $B$, then $G \backslash v$ is a quasi-block graph with $\operatorname{qc}(G \backslash v)=\operatorname{qc}(G)-1$.

Theorem 3.7. Let $G$ be a quasi-block graph. Then, $\operatorname{reg}\left(S / J_{G}\right) \leq c(G)$.

Proof. Let $G_{1}, \ldots, G_{c}$ be the connected components of $G$. Set $S_{G_{i}}=K\left[x_{j}, y_{j}: j \in V\left(G_{i}\right)\right]$. Then $S / J_{G} \cong S_{G_{1}} / J_{G_{1}} \otimes \cdots \otimes S_{G_{c}} / J_{G_{c}}$ which implies that $\operatorname{reg}\left(S / J_{G}\right)=\operatorname{reg}\left(S_{G_{1}} / J_{G_{1}}\right)+\cdots+$ $\operatorname{reg}\left(S_{G_{c}} / J_{G_{c}}\right)$. Also, $c(G)=c\left(G_{1}\right)+\cdots+c\left(G_{c}\right)$. Therefore, without loss of generality, we may assume that $G$ is a connected graph. We prove the result by induction on qc $(G)$. If $\operatorname{qc}(G)=0$, then every block of $G$ is a clique, thus $G$ is a block graph. By [7, Theorem 3.9], the assertion follows.

Assume that qc $(G)>0$. Let $B_{1}, \ldots, B_{\mathrm{qc}(G)}$ be the quasi-blocks of $G$. Now, we proceed by induction on the number of internal vertices of $B=B_{\mathrm{qc}(G)}$. It follows from Remark 3.3 that $\operatorname{iv}(B) \geq 2$. If $\operatorname{iv}(B)=2$, then there exists an edge $e$ such that $B \backslash e$ is a Cohen-Macaulay closed graph with exactly one internal vertex. Let $v \in V(B)$ be the internal vertex of $B \backslash e$. Therefore, $v$ is also an internal vertex of $G$. By Lemma 3.1, $J_{G}=J_{G_{v}} \cap\left(\left(x_{v}, y_{v}\right)+J_{G \backslash v}\right)$. Let $B_{v}$ be the block of $G_{v}$ which contains $v$. Note that $B_{v}$ is a clique as iv $(B)=2$. Since $v$ is an internal vertex of $B, v \notin V\left(B_{i}\right)$, for $i=1, \ldots, \mathrm{qc}(G)-1$ and hence, $\operatorname{qc}\left(G_{v}\right)=\operatorname{qc}(G)-1$. Therefore, by induction $\operatorname{reg}\left(S / J_{G_{v}}\right) \leq c\left(G_{v}\right)<c(G)$. It follows from Remark 3.6 and induction that $\operatorname{reg}\left(S /\left(\left(x_{v}, y_{v}\right)+J_{G \backslash v}\right)\right) \leq c(G \backslash v) \leq c(G)$. Since $G_{v} \backslash v$ is an induced subgraph of $G_{v}$, by [26, Proposition 8], $\operatorname{reg}\left(S /\left(\left(x_{v}, y_{v}\right)+J_{G_{v} \backslash v}\right)\right) \leq \operatorname{reg}\left(S / J_{G_{v}}\right)<c(G)$. Thus, it follows from Lemma 2.1 and the short exact sequence (11) that $\operatorname{reg}\left(S / J_{G}\right) \leq c(G)$.

Now, assume that $\operatorname{iv}(B)>2$. Let $v \in V(B)$ be an internal vertex of $B \backslash e$. Therefore, $v$ is an internal vertex of $G$. Again, by Lemma 3.1, $J_{G}=J_{G_{v}} \cap\left(\left(x_{v}, y_{v}\right)+J_{G \backslash v}\right)$. By Remark 3.6 and induction, $\operatorname{reg}\left(S /\left(\left(x_{v}, y_{v}\right)+J_{G \backslash v}\right)\right) \leq c(G \backslash v) \leq c(G)$. Let $B_{v}$ be the block of $G_{v}$ which contains $v$. If $\operatorname{iv}(B)=3$, then $B_{v}$ is a clique. Therefore, $\mathrm{qc}\left(G_{v}\right)=\mathrm{qc}(G)-1$ and hence, by induction $\operatorname{reg}\left(S / J_{G_{v}}\right) \leq c\left(G_{v}\right)<c(G)$. If $\operatorname{iv}(B)>3$, then $B_{v}$ is a quasi-block. By Lemma 3.2, $\operatorname{iv}\left(B_{v}\right)<\operatorname{iv}(B)$. Since $v$ is an internal vertex of $B, v \notin V\left(B_{i}\right)$, for $i=1, \ldots, \mathrm{qc}(G)-1$ and hence, $\mathrm{qc}\left(G_{v}\right)=\mathrm{qc}(G)$. Therefore, $G_{v}$ is a quasi-block graph with $\mathrm{qc}\left(G_{v}\right)=\mathrm{qc}(G)$ and $\operatorname{iv}\left(B_{v}\right)<\operatorname{iv}(B)$. By induction, $\operatorname{reg}\left(S / J_{G_{v}}\right) \leq c\left(G_{v}\right)<c(G)$, since $\operatorname{iv}\left(B_{v}\right)<\operatorname{iv}(B)$. It follows from [26, Proposition 8] that $\operatorname{reg}\left(S /\left(\left(x_{v}, y_{v}\right)+J_{G_{v} \backslash v}\right)\right) \leq \operatorname{reg}\left(S / J_{G_{v}}\right)<c(G)$ as $G_{v} \backslash v$ is an induced subgraph of $G_{v}$. Hence, using Lemma 2.1 in the short exact sequence (1), we conclude that $\operatorname{reg}\left(S / J_{G}\right) \leq c(G)$.

The following example illustrates that the upper bound obtained in Theorem 3.7 is tight.

Example 3.8. Let $G$ be a quasi-cycle graph such that for some $1 \leq i \leq s-1,\left|F_{i}\right|>2$ and $\left|F_{i+1}\right|>2$. Then $c(G)=l\left(G \backslash a_{i+1}\right) \leq l(G)$. Hence, it follows from [19, Theorem 1.1] and Theorem 3.7 that $\operatorname{reg}\left(S / J_{G}\right)=c(G)$. 
Also, the upper bound obtained in Theorem 3.7 can be strict upper bound. For example, $G=C_{n}$ is a quasi-block graph such that $c(G)=n$ and by [30, Corollary 16], $\operatorname{reg}\left(S / J_{G}\right)=$ $c(G)-2<c(G)$.

Let $H$ be a connected closed graph on $[m]$ such that $S_{H} / J_{H}$ is Cohen-Macaulay. By [5. Theorem 3.1], there exist integers $1=a_{1}<a_{2}<\cdots<a_{s}<a_{s+1}=m$ such that $F_{i}=\left[a_{i}, a_{i+1}\right]$, for $1 \leq i \leq s$ and $F_{1}, \ldots, F_{s}$ is a leaf order of $\Delta(H)$. Set $F_{s+1}=[m, n] \cup\{1\}$. The graph $G$ on the vertex set $[n]$ and edge set $E(G)=E(H) \cup\left\{\{i, j\}: i \neq j, i, j \in F_{s+1}\right\}$ is called a semi-cycle graph associated with $H$.

Example 3.9. Let $H$ and $G$ be the graphs shown in the figure below. Then, it can be seen that $H$ is a Cohen-Macaulay closed graph and $G$ is a semi-cycle graph associated with $H$.

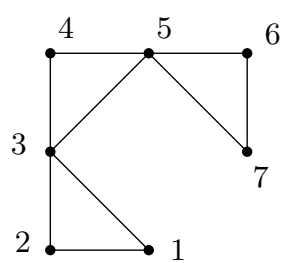

$H$

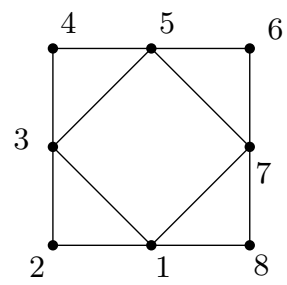

$G$

Remark 3.10. Let $G$ be a semi-cycle graph associated with the Cohen-Macaulay closed graph $H$. Let $F_{1}, \ldots, F_{s}$ be a leaf order of $\Delta(H)$. If $H \neq P_{3}$, then $\operatorname{iv}(G) \geq s$ and $\operatorname{iv}(H)=$ $s-1$. If $s=2$, then $G$ is a chordal graph. If $s>2$, then $G$ has an induced cycle on the vertex set $\left\{a_{1}, a_{2}, \ldots, a_{s}, a_{s+1}\right\}$ of length $s+1$ and hence, $G$ is not chordal. Note that every quasi-cycle is a semi-cycle, but a semi-cycle need not be a quasi-cycle. Also, $C_{n}$ is a semi-cycle graph.

A block $B$ of a graph $G$ is said to be a semi-block if $B$ is a semi-cycle with $B \neq K_{3}$. A graph $G$ is said to be a semi-block graph if all except one block are cliques and the block which is not a clique is a semi-block.

We now prove Conjecture 1.1 for semi-block graphs.

Theorem 3.11. Let $G$ be a semi-block graph. Then, $\operatorname{reg}\left(S / J_{G}\right) \leq c(G)$.

Proof. Let $B$ be the semi-block of $G$. If $B$ is a quasi-block of $G$, then $G$ is a quasi-block graph. Therefore, by Theorem 3.7, the assertion follows. Assume that $B$ is not a quasiblock, i.e., $\operatorname{iv}(B) \geq 3$ and for each $1 \leq i \leq s+1,\left|F_{i}\right| \geq 3$. We proceed by induction on $\operatorname{iv}(B)$. For $\operatorname{iv}(B)=3$, we claim that $\operatorname{reg}\left(S / J_{G}\right) \leq c(G)-1$. Let $v \in V(B)$ be an internal vertex of $B$. Therefore, $v$ is also an internal vertex of $G$. It follows from Lemma 3.1 that $J_{G}=J_{G_{v}} \cap\left(\left(x_{v}, y_{v}\right)+J_{G \backslash v}\right)$. Let $B_{v}$ be the block of $G_{v}$ which contains $v$. Note that $B_{v}$ is a chordal graph with $c\left(B_{v}\right)=2$ and hence, $G_{v}$ is a chordal graph with $c\left(G_{v}\right) \leq c(G)-2$. It follows from [24, Theorem 3.5] that $\operatorname{reg}\left(S / J_{G_{v}}\right) \leq c\left(G_{v}\right) \leq c(G)-2$. As $G \backslash v$ is a block graph with $c(G \backslash v) \leq c(G)-1$, by [7, Theorem 3.9], $\operatorname{reg}\left(S /\left(\left(x_{v}, y_{v}\right)+J_{G \backslash v}\right)\right) \leq$ 
$c(G \backslash v) \leq c(G)-1$. Since $G_{v} \backslash v$ is an induced subgraph of $G_{v}$, by [26, Proposition 8], $\operatorname{reg}\left(S /\left(\left(x_{v}, y_{v}\right)+J_{G_{v} \backslash v}\right)\right) \leq \operatorname{reg}\left(S / J_{G_{v}}\right)$. Thus, it follows from Lemma 2.1 and the short exact sequence (11) that $\operatorname{reg}\left(S / J_{G}\right) \leq c(G)-1$.

Assume that $\operatorname{iv}(B)>3$ and let $v$ be an internal vertex of $B$. Then, $G \backslash v$ is a block graph with $c(G \backslash v) \leq c(G)$. Therefore, by [7, Theorem 3.9], $\operatorname{reg}\left(S /\left(\left(x_{v}, y_{v}\right)+J_{G \backslash v}\right)\right) \leq c(G)$. Note that $B_{v}$ is a semi-block. It follows from Lemma 3.2 that $\operatorname{iv}\left(B_{v}\right)<\operatorname{iv}(B) . \operatorname{If} \operatorname{iv}(B)=4$, then $c\left(G_{v}\right) \leq c(G)$ and $\operatorname{iv}\left(B_{v}\right)=3$. Therefore, $\operatorname{reg}\left(S / J_{G_{v}}\right) \leq c\left(G_{v}\right)-1<c(G)$. If iv $(B)>4$, then $c\left(G_{v}\right)<c(G)$ and hence, by induction, $\operatorname{reg}\left(S / J_{G_{v}}\right) \leq c\left(G_{v}\right)<c(G)$. It follows from [26. Proposition 8] that $\operatorname{reg}\left(S /\left(\left(x_{v}, y_{v}\right)+J_{G_{v} \backslash v}\right)\right) \leq \operatorname{reg}\left(S / J_{G_{v}}\right)<c(G)$. Hence, by Lemma 2.1 and the short exact sequence(1) $\operatorname{reg}\left(S / J_{G}\right) \leq c(G)$.

For a chordal graph $G$, we recall a result from [24] which connects $c(G)$ and $c\left(G_{v}\right)$.

Lemma 3.12. [24, Lemma 3.4] Let $G$ be a chordal graph and $v$ be a vertex of $G$ which lies in $t$ maximal cliques of $G$. Then, $c\left(G_{v}\right) \leq c(G)-t+1$. In particular, if $t \geq 2$, then $c\left(G_{v}\right)<c(G)$.

As a consequence of Lemma 3.12, we have the following.

Corollary 3.13. Let $G$ be a chordal graph. If $v$ is an internal vertex of $G$, then $G_{v}$ is a chordal graph and $c\left(G_{v}\right)<c(G)$.

Proof. The assertion that $G_{v}$ is a chordal graph follows from the second paragraph of the proof of [24, Theorem 3.5]. Since $v$ is an internal vertex, $v$ belongs to at least two maximal cliques and hence, by Lemma 3.12, $c\left(G_{v}\right)<c(G)$.

Remark 3.14. If $G$ is a chordal graph, then $G \backslash v$ is a chordal graph for any $v$. If $v$ is an internal vertex of $G$, then $c(G \backslash v) \leq c(G)$.

In [24], Rouzbahani Malayeri et al. proved Saeedi Madani-Kiani conjecture for chordal graphs. They proved the result by induction on $n+c(G)$. We give another proof of the same by induction on $\operatorname{iv}(G)$.

Theorem 3.15. [24, Theorem 3.5] Let $G$ be a chordal graph. Then, $\operatorname{reg}\left(S / J_{G}\right) \leq c(G)$.

Proof. Without loss of generality, we may assume that $G$ is connected. We prove the assertion by induction on $\operatorname{iv}(G)$. If $\operatorname{iv}(G)=0$, then $G$ is a complete graph. Therefore, $c(G)=1$ and the result follows from Eagon-Northcott complex [4]. Assume that iv $(G)>0$ and if $H$ is a chordal graph with $\operatorname{iv}(H)<\operatorname{iv}(G)$, then $\operatorname{reg}\left(S_{H} / J_{H}\right) \leq c(H)$, where $S_{H}=K\left[x_{i}, y_{i}: i \in V(H)\right]$. Let $v$ be an internal vertex of $G$. It follows from Lemma 3.1 that $J_{G}=J_{G_{v}} \cap\left(\left(x_{v}, y_{v}\right)+J_{G \backslash v}\right)$.

By Corollary 3.13, $G_{v}$ is a connected chordal graph. Now, by Lemma 3.2, $\operatorname{iv}\left(G_{v}\right)<\operatorname{iv}(G)$ and hence, by induction, $\operatorname{reg}\left(S / J_{G_{v}}\right) \leq c\left(G_{v}\right)$.

It follows from Lemma 3.2 that $\operatorname{iv}(G \backslash v)<\operatorname{iv}(G)$. If $G \backslash v$ is connected, then by induction, $\operatorname{reg}\left(S /\left(\left(x_{v}, y_{v}\right)+J_{G \backslash v}\right)\right) \leq c(G \backslash v)$. If $v$ is a cut vertex, then let $H_{1}, \ldots, H_{p}$ be the connected 
components of $G \backslash v$. By induction, $\operatorname{reg}\left(S_{H_{i}} / J_{H_{i}}\right) \leq c\left(H_{i}\right)$ for each $i$. Therefore,

$$
\operatorname{reg}\left(S /\left(\left(x_{v}, y_{v}\right)+J_{G \backslash v}\right)\right)=\sum_{i \in[p]} \operatorname{reg}\left(S_{H_{i}} / J_{H_{i}}\right) \leq \sum_{i \in[p]} c\left(H_{i}\right)=c(G \backslash v) .
$$

The graph $G_{v} \backslash v$ is an induced subgraph of $G_{v}$. Therefore, by [26, Proposition 8], $\operatorname{reg}\left(S /\left(\left(x_{v}, y_{v}\right)+J_{G_{v} \backslash v}\right)\right) \leq \operatorname{reg}\left(S / J_{G_{v}}\right) \leq c\left(G_{v}\right)$.

Now, by Corollary 3.13, $c\left(G_{v}\right)<c(G)$ and by Remark 3.14, $c(G \backslash v) \leq c(G)$. Hence, by applying Lemma 2.1 in the short exact sequence (1), we get the desired result.

We recall notation of decomposability from [23]. A graph $G$ is called decomposable, if there exist subgraphs $G_{1}$ and $G_{2}$ such that $G$ is obtained by identifying a free vertex $v_{1}$ of $G_{1}$ with a free vertex $v_{2}$ of $G_{2}$, i.e., $G=G_{1} \cup G_{2}$ with $V\left(G_{1}\right) \cap V\left(G_{2}\right)=\{v\}$ such that $v$ is a free vertex of both $G_{1}$ and $G_{2}$.

A graph $G$ is called indecomposable, if it is not decomposable. Up to ordering, $G$ has a unique decomposition into indecomposable subgraphs, i.e., there exist $G_{1}, \ldots, G_{r}$ indecomposable induced subgraphs of $G$ with $G=G_{1} \cup \cdots \cup G_{r}$ such that for each $i \neq j$, either $V\left(G_{i}\right) \cap V\left(G_{j}\right)=\emptyset$ or $V\left(G_{i}\right) \cap V\left(G_{j}\right)=\{v\}$ and $v$ is a free vertex of both $G_{i}$ and $G_{j}$.

It follows from [13, Theorem 3.1] that if $G=G_{1} \cup \cdots \cup G_{r}$ is a decomposition into indecomposable graphs, then $\operatorname{reg}\left(S / J_{G}\right)=\sum_{i=1}^{r} \operatorname{reg}\left(S / J_{G_{i}}\right)$. Therefore, we consider indecomposable graphs to study the regularity.

Let $u, v \in V(G)$ be such that $e=\{u, v\} \notin E(G)$, then we denote by $G_{e}$, the graph on the vertex set $V(G)$ and edge set $E\left(G_{e}\right)=E(G) \cup\left\{\{x, y\}: x, y \in N_{G}(u)\right.$ or $\left.x, y \in N_{G}(v)\right\}$. An edge $e$ is said to be a cut edge if the number of connected components of $G \backslash e$ is more than the number of connected components of $G$.

In the following theorem, we give a sufficient condition for a chordal graph whose regularity is not maximal.

Theorem 3.16. Let $G$ be a connected indecomposable chordal graph on $[n]$. If $G$ has a cut edge, then $\operatorname{reg}\left(S / J_{G}\right)<c(G)$.

Proof. Let $e=\{u, v\}$ be a cut edge of $G$. Let $H_{1}$ and $H_{2}$ be connected components of $G \backslash e$ with $u \in V\left(H_{1}\right)$ and $v \in V\left(H_{2}\right)$. Since $G$ is an indecomposable graph, $u$ and $v$ are internal vertices of $H_{1}$ and $H_{2}$, respectively. Note that $(G \backslash e)_{e}=\left(H_{1}\right)_{u} \sqcup\left(H_{2}\right)_{v}$. By Corollary 3.13, $\left(H_{1}\right)_{u}$ and $\left(H_{2}\right)_{v}$ are chordal graphs and $c\left((G \backslash e)_{e}\right)=c\left(\left(H_{1}\right)_{u}\right)+c\left(\left(H_{2}\right)_{v}\right) \leq$ $c\left(H_{1}\right)+c\left(H_{2}\right)-2=c(G)-3$. Therefore, by Theorem 3.15, $\operatorname{reg}\left(S / J_{(G \backslash e)_{e}}\right)=\operatorname{reg}\left(S_{H_{1}} / J_{\left(H_{1}\right)_{u}}\right)+$ $\operatorname{reg}\left(S_{H_{2}} / J_{\left(H_{2}\right)_{v}}\right) \leq c(G)-3$. Also, $G \backslash e=H_{1} \sqcup H_{2}$ is a chordal graph and $c(G \backslash e)=c(G)-1$. Therefore, by Theorem 3.15, $\operatorname{reg}\left(S / J_{G \backslash e}\right)=\operatorname{reg}\left(S_{H_{1}} / J_{H_{1}}\right)+\operatorname{reg}\left(S_{H_{2}} / J_{H_{2}}\right) \leq c(G)-1$. It follows from Lemma [15, Proposition 2.1(a)] that $\operatorname{reg}\left(S / J_{G}\right)<c(G)$.

The following example illustrates that the assumption in Theorem 3.16, that $G$ contains a cut edge, is not a necessary condition. First, we recall the definition of join of graphs. Let $H$ and $H^{\prime}$ be two graphs with the vertex sets $[p]$ and $[q]$, respectively. The join of 
$H$ and $H^{\prime}$, denoted by $H * H^{\prime}$, is the graph with vertex set $[p] \sqcup[q]$ and the edge set $E\left(H * H^{\prime}\right)=E(H) \cup E\left(H^{\prime}\right) \cup\{\{i, j\} \mid i \in[p], j \in[q]\}$.

Example 3.17. For $n \geq 3$, let $G=P_{2} * K_{n}^{c}$, where $K_{n}^{c}$ is a graph on $n$ vertices and has no edges. Note that $G$ is an indecomposable chordal graph on $n+2$ vertices. Observe that $c(G)=n$ and $G$ has no cut edge. It follows from [27, Theorem 2.1] that $\operatorname{reg}\left(S / J_{G}\right)=$ $\max \left\{\operatorname{reg}\left(S / J_{P_{2}}\right), 2\right\}=2$.

\section{Regularity of BinOmial EDGe ideAls of Jahangir GRAPHS}

In this section, we obtain the regularity of binomial edge ideals of Jahangir graphs. First, we recall the definition of Jahangir graph.

Definition 4.1. The Jahangir graph denoted by $J_{m, n}$ is a graph on the vertex set $[m n+1]$, for $m \geq 1$ and $n \geq 3$, such that the induced subgraph on $[m n]$ is $C_{m n}$ and the neighborhood of vertex $m n+1$ is $\{1, m+1, \ldots, m(n-1)+1\}$.

Example 4.2. We give illustrations of $J_{1,8}$ and $J_{2,4}$ below.

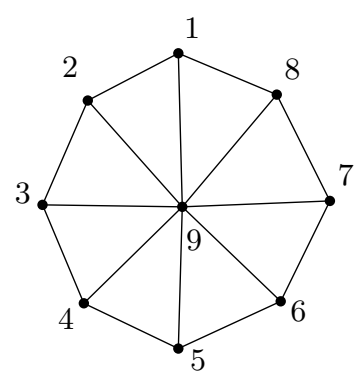

$J_{1,8}$

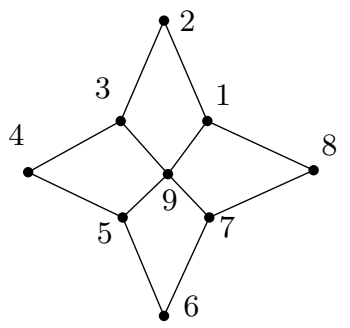

$J_{2,4}$

One can note that if $m>1$, then $J_{m, n}$ is not a chordal graph and $c\left(J_{m, n}\right)=n(m+1)$. It follows from [15, Theorem 3.2] that

$$
\operatorname{reg}\left(S / J_{J_{m, n}}\right) \leq m n-1<c\left(J_{m, n}\right) .
$$

Since $l\left(J_{m, n}\right)=m n-2$, by [19, Theorem 1.1], $m n-2 \leq \operatorname{reg}\left(S / J_{J_{m, n}}\right)$. If $m=1$, then $J_{1, n}$ is the wheel graph $W_{n}$ and hence, by virtue of [27, Theorem 2.1], $\operatorname{reg}\left(S / J_{J_{1, n}}\right)=n-2$. We now prove the same for $m \geq 2$. First, we recall a result due to Ene et al. from [6] which is useful to compute the regularity of binomial edge ideals of Jahangir graphs.

Theorem 4.3. [6, Theorem 2.1] Let $G$ be a connected graph on $[n]$. Then, $\operatorname{reg}\left(S / J_{G}\right) \leq$ $n-\omega(G)+1$.

We now obtain the regularity of binomial edge ideals of Jahangir graphs. To compute the regularity of $J_{m, n}$, we need the following lemma. The idea of this lemma is based on [6, Lemma 3.3]. 
Lemma 4.4. Let $G$ be a connected graph on $[n]$. If $G$ has an internal vertex $v$ such that $\operatorname{deg}_{G}(v) \geq 4$ and $G \backslash v$ is not a path graph on $n-1$ vertices, then $\operatorname{reg}\left(S / J_{G}\right) \leq n-3$.

Proof. Since $v$ is an internal vertex, by Lemma 3.1, $J_{G}=J_{G_{v}} \cap\left(\left(x_{v}, y_{v}\right)+J_{G \backslash v}\right)$. Note that

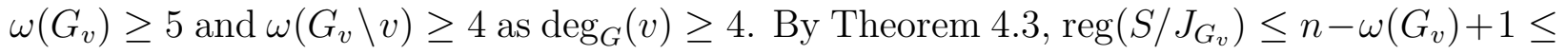
$n-4$ and $\operatorname{reg}\left(S /\left(\left(x_{v}, y_{v}\right)+J_{G_{v} \backslash v}\right)\right) \leq(n-1)-\omega\left(G_{v} \backslash v\right)+1 \leq n-4$. Since $G \backslash v$ is not a path graph, it follows from [15, Theorem 3.2] that $\operatorname{reg}\left(S /\left(\left(x_{v}, y_{v}\right)+J_{G \backslash v}\right)\right) \leq(n-1)-2=n-3$. Hence, the assertion follows from Lemma 2.1 and the short exact sequence (11).

We conclude this section by computing the regularity of binomial edge ideals of Jahangir graphs.

Theorem 4.5. For $m \geq 2$ and $n \geq 3$, let $G=J_{m, n}$. Then, $\operatorname{reg}\left(S / J_{G}\right)=m n-2$.

Proof. Since $l(G)=m n-2$, by [19, Theorem 1.1], $m n-2 \leq \operatorname{reg}\left(S / J_{G}\right)$. Therefore, it is enough to prove that $\operatorname{reg}\left(S / J_{G}\right) \leq m n-2$. Set $v=m n+1$. Observe that $v$ is an internal vertex of $G$. If $n \geq 4$, then $\operatorname{deg}_{G}(v)=n \geq 4$ and $G \backslash v$ is not a path graph on $m n$ vertices. Thus, by Lemma 4.4, $\operatorname{reg}\left(S / J_{G}\right) \leq m n-2$.

Now, we assume that $n=3$. By Lemma 3.1, $J_{G}=J_{G_{v}} \cap\left(\left(x_{v}, y_{v}\right)+J_{G \backslash v}\right)$. Note that $\omega\left(G_{v}\right)=4$. Hence, by virtue of Theorem 4.3, $\operatorname{reg}\left(S / J_{G_{v}}\right) \leq(3 m+1)-4+1=3 m-3$. Set $H=G_{v} \backslash v$. Then, $H$ is a connected graph on $3 m$ vertices, $\operatorname{deg}_{H}(1)=4$ and $H \backslash 1$ is not a path graph on $3 m-1$ vertices. Thus, by Lemma 4.4 $\operatorname{reg}\left(S /\left(\left(x_{v}, y_{v}\right)+J_{H}\right)\right) \leq 3 m-3$. It is clear that $G \backslash v=C_{3 m}$, thus by [30, Corollary 16], $\operatorname{reg}\left(S /\left(\left(x_{v}, y_{v}\right)+J_{G \backslash v}\right)\right)=3 m-2$. By virtue of Lemma 2.1 and the short exact sequence (1) , we get $\operatorname{reg}\left(S / J_{G}\right) \leq 3 m-2$.

\section{Hibi-Matsuda Conjecture}

Let $M=\bigoplus_{k \in \mathbb{N}} M_{k}$ be a finite graded $S$-module of Krull dimension $d$. The function $H_{M}: \mathbb{N} \rightarrow$ $\mathbb{N}$ defined as $H_{M}(k)=l\left(M_{k}\right)$ is called the Hilbert function of $M$. The Hilbert series of $M$ is the generating series of the Hilbert function $H_{M}$ and is denoted by $\operatorname{Hilb}_{M}(t)=\sum_{k \in \mathbb{N}} l\left(M_{k}\right) t^{k}$. By [2, Corollary 4.1.8], there exists a unique polynomial $h_{M}(t) \in \mathbb{Z}[t]$ such that $h_{M}(1) \neq 0$ and $\operatorname{Hilb}_{M}(t)=h_{M}(t) /(1-t)^{d}$. The polynomial $h_{M}(t)$ is called the $h$-polynomial of $M$.

In this section, we give a sufficient condition for Conjecture 1.2 to be true. We prove that Conjecture 1.2 holds if $S / J_{G}$ admits a unique extremal Betti number. In [29], Schenzel and Zafar proved that complete bipartite graphs have unique extremal Betti number. Zafar and Zahid proved that the $n$-cycle, $C_{n}$, has a unique extremal Betti number, see [30]. In [10], Herzog and Rinaldo characterized block graphs which admit unique extremal Betti number. In [16], we have characterized generalized block graphs which admit unique extremal Betti number.

Theorem 5.1. Let $G$ be a connected graph on $[n]$. If $S / J_{G}$ admits a unique extremal Betti number, then $\operatorname{reg}\left(S / J_{G}\right) \leq \operatorname{deg} h_{S / J_{G}}(t)$. 
Proof. Set $p=\operatorname{pd}\left(S / J_{G}\right)$ and $r=\operatorname{reg}\left(S / J_{G}\right)$. If $G$ is not a complete graph, then by [1, Theorem B], $p \geq n+\kappa(G)-2$, where $\kappa(G)$ is the vertex connectivity of $G$. If $G$ is a complete graph, then $p=n-1$. Note that for any connected graph $G$ if $G \neq K_{n}$, then $\kappa(G) \geq 1$. Therefore, for any connected graph $G, p \geq n-1$. Also, it follows from [9, Corollary 3.3] that $\operatorname{dim}\left(S / J_{G}\right) \geq n+1$. Now, by [2, Corollary 4.1.14],

$$
\sum_{i, j}(-1)^{i} \beta_{i, j}^{S}\left(S / J_{G}\right) t^{j}=h_{S / J_{G}}(t)(1-t)^{2 n-d}
$$

where $d=\operatorname{dim}\left(S / J_{G}\right)$. Since $S / J_{G}$ has the unique extremal Betti number $\beta_{p, p+r}^{S}\left(S / J_{G}\right)$, we get $p+r=2 n-d+\operatorname{deg} h_{S / J_{G}}(t)$. Hence, the assertion follows.

It is natural to ask if Conjecture 1.2 is true when $S / J_{G}$ has more than one extremal Betti numbers. Here is one instance when $S / J_{G}$ has two extremal Betti number and Conjecture 1.2 is true. A flower graph $F_{h, k}(v)$ is a connected graph obtained by identifying a free vertex as $v$, each of $h$ copies of the complete graph $K_{3}$ and $k$ copies of the star graph $K_{1,3}$ with $h+k \geq 3$. The flower graph was introduced by Mascia and Rinaldo in [18]. It follows from [18, Theorem 3.4] that $F_{h, k}(v)$ has two extremal Betti numbers.

Theorem 5.2. Let $G=F_{h, k}(v)$ be a flower graph. Then, $\operatorname{reg}\left(S / J_{G}\right) \leq \operatorname{deg} h_{S / J_{G}}(t)$.

Proof. It follows from [2, Corollary 4.1.14] that

$$
\sum_{i, j}(-1)^{i} \beta_{i, j}\left(S / J_{G}\right) t^{j}=h_{S / J_{G}}(t)(1-t)^{2 n-d}
$$

where $d=\operatorname{dim}\left(S / J_{G}\right)$. By [18, Theorem 3.4], the degree of the polynomial on the left hand side of the above equation is $n+\mathrm{iv}(G)$. Therefore, by comparing the degree, we get $n+\operatorname{iv}(G)=2 n-d+\operatorname{deg} h_{S / J_{G}}(t)$. It follows from [18, Corollary 3.5] that $\operatorname{reg}\left(S / J_{G}\right)=\operatorname{iv}(G)+$ $\operatorname{cdeg}(v)-1=n-d+\operatorname{deg} h_{S / J_{G}}(t)+\operatorname{cdeg}(v)-1$. Let $T=\{v\}$. Note that, $c_{G}(T)=\operatorname{cdeg}(v)$. By [9, Corollary 3.3], $d \geq n+\operatorname{cdeg}(v)-1$. Thus, we get $\operatorname{reg}\left(S / J_{G}\right) \leq \operatorname{deg} h_{S / J_{G}}(t)$.

Now, we provide a counterexample to Hibi-Matsuda conjecture. In [14], Kahle and Krüsemann gave a counterexample to Hibi-Matsuda conjecture. However, one can observe that the counterexample given in [14] is not a chordal graph. Here, we provide a counterexample which is a block graph and hence, a chordal graph.

Example 5.3. The following graph is a counterexample to Hibi-Matsuda Conjecture. 


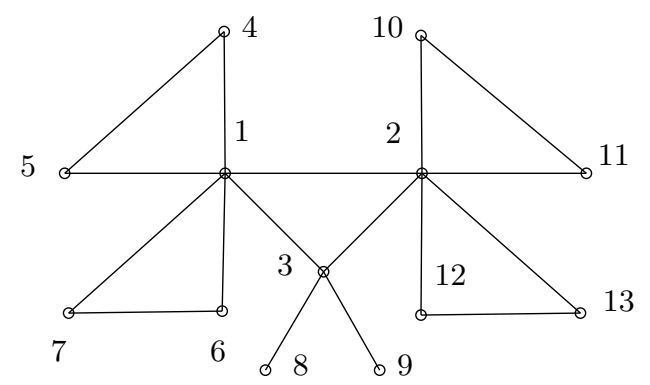

$G$

It follows from [18, Theorem 4.2] that $\operatorname{reg}\left(S / J_{G}\right)=6$. We computed the Hilbert series of $S / J_{G}$ using Macaulay 2 package [8]:

$$
\operatorname{Hilb}_{S / J_{G}}(t)=\frac{1+10 t+38 t^{2}+60 t^{3}+19 t^{4}-24 t^{5}}{(1-t)^{18}} .
$$

The polynomial $h_{S / J_{G}}(t)=1+10 t+38 t^{2}+60 t^{3}+19 t^{4}-24 t^{5}$ is the $h$-polynomial of $S / J_{G}$ and $\operatorname{deg} h_{S / J_{G}}(t)=5<\operatorname{reg}\left(S / J_{G}\right)$. Let $G_{1}, \ldots, G_{k}$ be $k$ copies of the graph $G$. The graph $G^{k}$ is obtained by identifying a free vertex of $G_{i}$ with a free vertex of $G_{i+1}$, i.e. $G^{k}=G_{1} \cup \cdots \cup G_{k}$, $V\left(G_{i}\right) \cap V\left(G_{j}\right)=\emptyset$, if $j \notin\{i-1, i+1\}$ and $V\left(G^{i}\right) \cap V\left(G^{i+1}\right)=\left\{u_{i}\right\}$, where $u_{i}$ is a free vertex of $G^{i}$ and $G^{i+1}$. Then, it follows from [13, Theorem 3.1] that $\operatorname{reg}\left(S_{k} / J_{G^{k}}\right)=6 k$, where $S_{k}=K\left[x_{i}, y_{i}: i \in V\left(G^{k}\right)\right]$. Also, by [17, Corollary 3.3], $\operatorname{deg} h_{S_{k} / J_{G}}(t)=5 k$. This shows that for any positive integer $k$, there is a graph $G^{k} \operatorname{such}$ that $\operatorname{reg}\left(S_{k} / J_{G^{k}}\right)-\operatorname{deg} h_{S_{k} / J_{G}}(t)=k$.

In [3], de Alba and Hoang asked whether the initial ideal of the binomial edge ideal of a closed graph admits a unique extremal Betti number, (see [3, Question 1]). Recently, in [28], Saeedi Madani and Kiani gave a negative answer to the above question, (see [28, Theorem 4.6]). We end this article by asking the following question.

Question 5.4. When does the binomial edge ideal of a graph admit a unique extremal Betti number?

Acknowledgements: The author is grateful to his advisor A. V. Jayanthan for his constant support, valuable ideas and suggestions. The author thanks the National Board for Higher Mathematics, India for the financial support. The author also wishes to express his sincere gratitude to the anonymous referees whose comments helped improve the exposition in great detail.

\section{REFERENCES}

[1] Arindam Banerjee and Luis Núñez Betancourt. Graph connectivity and binomial edge ideals. Proc. Amer. Math. Soc., 145(2):487-499, 2017.

[2] Winfried Bruns and Jürgen Herzog. Cohen-Macaulay rings, volume 39 of Cambridge Studies in Advanced Mathematics. Cambridge University Press, Cambridge, 1993. 
[3] Hernán de Alba and Do Trong Hoang. On the extremal Betti numbers of the binomial edge ideal of closed graphs. Math. Nachr., 291(1):28-40, 2018.

[4] J. A. Eagon and D. G. Northcott. Ideals defined by matrices and a certain complex associated with them. Proc. Roy. Soc. Ser. A, 269:188-204, 1962.

[5] Viviana Ene, Jürgen Herzog, and Takayuki Hibi. Cohen-Macaulay binomial edge ideals. Nagoya Math. J., 204:57-68, 2011.

[6] Viviana Ene, Giancarlo Rinaldo, and Naoki Terai. Licci binomial edge ideals. arXiv e-prints, page arXiv:1910.03612, Oct 2019.

[7] Viviana Ene and Andrei Zarojanu. On the regularity of binomial edge ideals. Math. Nachr., 288(1):19$24,2015$.

[8] Daniel R. Grayson and Michael E. Stillman. Macaulay2, a software system for research in algebraic geometry. Available at http://www.math.uiuc.edu/Macaulay2/

[9] Jürgen Herzog, Takayuki Hibi, Freyja Hreinsdóttir, Thomas Kahle, and Johannes Rauh. Binomial edge ideals and conditional independence statements. Adv. in Appl. Math., 45(3):317-333, 2010.

[10] Jürgen Herzog and Giancarlo Rinaldo. On the extremal Betti numbers of binomial edge ideals of block graphs. Electron. J. Combin., 25(1):Paper 1.63, 10, 2018.

[11] Takayuki Hibi and Kazunori Matsuda. Regularity and h-polynomials of binomial edge idals. arXiv e-prints, page arXiv:1808.06984, Aug 2018.

[12] A. V. Jayanthan and Arvind Kumar. Regularity of binomial edge ideals of Cohen-Macaulay bipartite graphs. Comm. Algebra, 47(11):4797-4805, 2019.

[13] A. V. Jayanthan, N. Narayanan, and B. V. Raghavendra Rao. Regularity of binomial edge ideals of certain block graphs. Proc. Indian Acad. Sci. Math. Sci., 129(3):Art. 36, 10, 2019.

[14] Thomas Kahle and Jonas Krüsemann. Binomial edge ideals of cographs. arXiv e-prints, page arXiv:1906.05510, Jun 2019.

[15] Dariush Kiani and Sara Saeedi Madani. The Castelnuovo-Mumford regularity of binomial edge ideals. J. Combin. Theory Ser. A, 139:80-86, 2016.

[16] Arvind Kumar. Binomial Edge Ideal of Generalized block graph. arXiv e-prints, page arXiv:1910.06787, Oct 2019.

[17] Arvind Kumar and Rajib Sarkar. Hilbert series of binomial edge ideals. Comm. Algebra, 47(9):3830$3841,2019$.

[18] Carla Mascia and Giancarlo Rinaldo. Krull dimension and regularity of binomial edge ideals of block graphs. Journal of Algebra and Its Applications, To Appear.

[19] Kazunori Matsuda and Satoshi Murai. Regularity bounds for binomial edge ideals. J. Commut. Algebra, $5(1): 141-149,2013$.

[20] Fatemeh Mohammadi and Leila Sharifan. Hilbert function of binomial edge ideals. Comm. Algebra, 42(2):688-703, 2014.

[21] Masahiro Ohtani. Graphs and ideals generated by some 2-minors. Comm. Algebra, 39(3):905-917, 2011.

[22] Irena Peeva. Graded syzygies, volume 14 of Algebra and Applications. Springer-Verlag London, Ltd., London, 2011.

[23] Asia Rauf and Giancarlo Rinaldo. Construction of Cohen-Macaulay binomial edge ideals. Comm. Algebra, 42(1):238-252, 2014.

[24] M. Rouzbahani Malayeri, S. Saeedi Madani, and D. Kiani. Regularity of binomial edge ideals of chordal graphs. arXiv e-prints, page arXiv:1810.03119, Oct 2018.

[25] Sara Saeedi Madani and Dariush Kiani. Binomial edge ideals of graphs. Electron. J. Combin., 19(2):Paper 44, 6, 2012. 
[26] Sara Saeedi Madani and Dariush Kiani. On the binomial edge ideal of a pair of graphs. Electron. J. Combin., 20(1):Paper 48, 13, 2013.

[27] Sara Saeedi Madani and Dariush Kiani. Binomial edge ideals of regularity 3. J. Algebra, 515:157-172, 2018.

[28] Sara Saeedi Madani and Dariush Kiani. Induced matchings in strongly biconvex graphs and some algebraic applications. arXiv e-prints, page arXiv:1905.02640, May 2019.

[29] Peter Schenzel and Sohail Zafar. Algebraic properties of the binomial edge ideal of a complete bipartite graph. An. Ştiinţ. Univ. "Ovidius” Constanţa Ser. Mat., 22(2):217-237, 2014.

[30] Sohail Zafar and Zohaib Zahid. On the Betti numbers of some classes of binomial edge ideals. Electron. J. Combin., 20(4):Paper 37, 14, 2013.

Email address: arvkumar11@gmail.com

Department of Mathematics, Indian Institute of Technology Madras, Chennai, 600036, INDIA 